\title{
BMJ Open Role of diffusional kurtosis imaging in grading of brain gliomas: a protocol for systematic review and meta-analysis
}

\author{
Gehad Abdalla, ${ }^{1}$ Eser Sanverdi, ${ }^{1}$ Pedro M Machado, ${ }^{2}$ Joey S W Kwong, ${ }^{3}$ \\ Jasmina Panovska-Griffiths, ${ }^{4,5}$ Antonio Rojas-Garcia, ${ }^{4}$ Daisuke Yoneoka, ${ }^{6}$ \\ Tarek Yousry, ${ }^{1,7}$ Sotirios Bisdas ${ }^{1,7}$
}

To cite: Abdalla G, Sanverdi E, Machado PM, et al. Role of diffusional kurtosis imaging in grading of brain gliomas: a protocol for systematic review and meta-analysis. BMJ Open 2018;8:e025123. doi:10.1136/ bmjopen-2018-025123

- Prepublication history for this paper is available online. To view these files please visit the journal online (http://dx.doi. org/10.1136/bmjopen-2018025123).

Received 30 June 2018 Revised 4 October 2018 Accepted 9 October 2018
Check for updates

(C) Author(s) (or their employer(s)) 2018. Re-use permitted under CC BY-NC. No commercial re-use. See rights and permissions. Published by BMJ.

For numbered affiliations see end of article.

Correspondence to Professor Sotirios Bisdas; s.bisdas@ucl.ac.uk

\section{ABSTRACT}

Introduction Central nervous system (CNS) gliomas are the most common primary intra-axial brain tumours and pose variable treatment response according to their grade, therefore, precise staging is mandatory. Histopathological analysis of surgical tumour samples is still deemed as the state-of-the-art staging technique for gliomas due to the moderate specificity of the available non-invasive imaging modalities. A recently evolved analysis of the tissue water diffusion properties, known as diffusional kurtosis imaging (DKI), is a dimensionless metric, which quantifies water molecules' degree of non-Gaussian diffusion, hence reflects tissue microenvironment's complexity by means of non-invasive diffusion-weighted MRI acquisitions. The objective of this systematic review and meta-analysis is to explore the performance of DKI in the presurgical grading of gliomas, both regarding the differentiation between high-grade and low-grade gliomas as well as the discrimination between gliomas and other intra-axial brain tumours.

Methods and analysis We will search PubMed, Medline via Ovid, Embase and Scopus in July 2018 for research studies published between January 1990 and June 2018 with no language restrictions, which have reported on the performance of DKI in diagnosing CNS gliomas. Robust inclusion/exclusion criteria will be applied for selection of eligible articles. Two authors will separately perform quality assessment according to the quality assessment of diagnostic accuracy studies-2 tool. Data will be extracted in a predesigned spreadsheet. A meta-analysis will be held using a random-effects model if substantial statistical heterogeneity is expected. The heterogeneity of studies will be evaluated, and sensitivity analyses will be conducted according to individual study quality.

Ethics and dissemination This work will be based on published studies; hence, it does not require institutional review board approval or ethics clearance. The results will be published in peer-reviewed journals.

PROSPERO registration number CRD42018099192.

\section{INTRODUCTION}

Gliomas are the most common primary intra-axial brain tumours. Precise and robust diagnosis and staging are crucial for implementing successful management strategies and predicting their outcome. However, there

\section{Strengths and limitations of this study}

- The findings of this review and meta-analysis will contribute to implementing diffusional kurtosis imaging (DKI) in the routine MRI assessment and staging of intra-axial brain tumours and gliomas, respectively.

- The conducted meta-analysis will provide evidence on the diagnostic performance of the technique which will help to establish a universally accepted standardised DKI imaging protocol.

- The quality assessment of the studies will follow the modified quality assessment of diagnostic accuracy studies-2 tool offering an objective method for evaluating risk of bias in the studies.

- This study will strongly follow the Preferred Reporting Items for Systematic Reviews and Meta-Analyses of Diagnostic Test Accuracy Studies checklist.

- The limitation of this work will be any heterogeneity among the included studies in image acquisition techniques, postprocessing image analysis methodologies and variability in the extracted DKI metrics.

is noticeable variation in individual tumour response even if they are attributed the same histological grade. This could be explained by the divergent proliferative patterns, cellular complexity and neovascularisation among these tumours. ${ }^{1}$ WHO 2007 classification used to classify brain gliomas into four subgroups depending on their histopathological features such as cellular atypia, mitotic activity and anaplasia. ${ }^{2}$ Meanwhile, efforts have been made in order to further stratify gliomas based on their molecular features aiming to better explain the tumours' behaviour and determine better treatment strategies. The revised fourth edition of the updated WHO classification of central nervous system tumours in 2016 incorporates both molecular and histopathological features of gliomas. ${ }^{3}$ As previously, histopathological examination after neurosurgical sampling is still considered as the state-ofthe-art staging method of gliomas; owing to the 


\begin{tabular}{ll}
\hline Table 1 & Search Syntax in PubMed and Medline \\
\hline Database & Search syntax \\
\hline PubMed & ("Glioma"[Mesh] OR "Brain Neoplasms"[Mesh] \\
& OR glioma[TW] OR gliomas[TW] OR (brain[TW] \\
& AND neoplasm*[TW])) AND ((diffusion[TW] \\
& AND kurtosis[TW]) OR (diffusional[tw] \\
& AND kurtosis[TW]) OR DKI[TW] OR "non- \\
& Gaussian"[TW]) \\
Medline & 1. diffusional kurtosis.mp. \\
& 2. diffusion kurtosis.mp. \\
& 3. DKI.mp. \\
4. non gaussian.mp. & 5. 1 or 2 or 3 or 4 \\
6. exp Glioma/ \\
7. exp Brain Neoplasms/ \\
8. (glioma or gliomas or brain neoplasm*). \\
mp. [mp=title, abstract, original title, name \\
of substance word, subject heading word, \\
keyword heading word, protocol supplementary \\
concept word, rare disease supplementary \\
concept word, unique identifier, synonyms] \\
9. 6 or 7 or 8 \\
10. 5 and 9 \\
\end{tabular}

moderate specificity of non-invasive imaging techniques. ${ }^{45}$ On the other hand, there is a demand for reliable non-invasive imaging methods, namely for patients who are unfit for surgery, to elucidate concerns regarding postbiopsy complications, or even to diagnose patients with suspected low-grade gliomas whose management can be regular follow-up. ${ }^{3}$ In the last few years, both diffusion-weighted imaging (DWI) and diffusion tensor imaging (DTI) have emerged as presurgical imaging tools and as tools for evaluating treatment response to chemotherapy and radiotherapy regimens in patients with gliomas. ${ }^{6}$ However, DWI and DTI quantify water molecules' mobility on the assumption of unrestricted-but possibly hindered-random diffusion. Accordingly, the likelihood of certain proton diffusing from one location to another in a given time (known as the probability distribution function, PDF) is thought to be Gaussian related. ${ }^{7}$ However, the paramount differences inside the brain tissue cytoarchitecture due to cell membranes, organelles and discrete compartments will drift the diffusion of water molecules from the normal Gaussian distribution. Therefore, the real PDF will be more soared up in contrast to the Gaussian PDF. The widely used metric of diffusion attenuation in tumours, namely the apparent diffusion coefficient, is limited in detecting this deviation from the normal Gaussian behaviour. A novel diffusion model known as diffusional kurtosis imaging (DKI) is a dimensionless metric that has recently evolved to quantify water molecules' degree of non-Gaussianity. ${ }^{17}$ Thus, DKI can provide a more realistic biomarker that reflects the brain microenvironment's complexity. ${ }^{8}$ It is simply considered a continuation of the DTI model and at least 2 non-zero diffusion gradient factors (b-values) in more than 15 non-linear diffusion directions are applied to acquire both the kurtosis metrics (radial kurtosis, axial kurtosis and mean kurtosis $(\mathrm{Mk})$ ) and the diffusion tensor metrics (mean diffusivity and fractional anisotropy simultaneously. ${ }^{1}$ It is therefore important to understand the diagnostic performance of DKI, both regarding its ability to stage the gliomas in subgroups and to differentiate gliomas from other intra-axial brain tumours.

Recent studies have shown promising results. ${ }^{89}$ In April 2018, a systematic review and meta-analysis of the diagnostic accuracy of DKI for glioma grading revealed that the pooled area under the curve for Mk in differentiating high-grade from low-grade gliomas was $0.94 .{ }^{10}$ This review included only 10 studies, whereas in the current work, we believe that more studies about DKI have been held in the interim. Moreover, we aim to include studies that compared DKI between gliomas and other intra-axial brain tumours.

\section{OBJECTIVE}

This review aims to investigate the diagnostic performance of DKI in the grading of gliomas and the differentiation between gliomas and other intra-axial brain tumours.

\section{REVIEW QUESTIONS}

How accurate is DKI in differentiating between highgrade and low-grade gliomas and in differentiating brain gliomas from other intra-axial tumours?

\section{METHODS}

This review protocol was developed according to the Preferred Reporting Items for Systematic Reviews and Meta-Analyses-Protocols (PRISMA) statement and guidance from the Joanna Briggs Institute Reviewers' Manual for the systematic review of studies of diagnostic test accuracy. ${ }^{11} 12$ The subsequent full systematic review will be prepared according to the PRISMA-Diagnostic Test Accuracy Studies checklist. ${ }^{13}$

\section{Patient and public involvement}

This work will be based on published studies; hence, there will not be patient or public involvement. The results will be published in peer-reviewed journals.

\section{Inclusion and exclusion criteria}

We will include all studies that have investigated DKI in tumours of glial cell origin, either primary or recurrent gliomas, in adult patients, using either WHO 2007 or WHO 2016 classifications and compared the DKI features in gliomas with other non-glial tumours. Exclusion criteria will comprise paediatric groups, non-original research articles (reviews, commentaries, erratum, books, editorial and conference abstracts), animal studies, non-imaging studies, non-MRI studies, non-DKI 


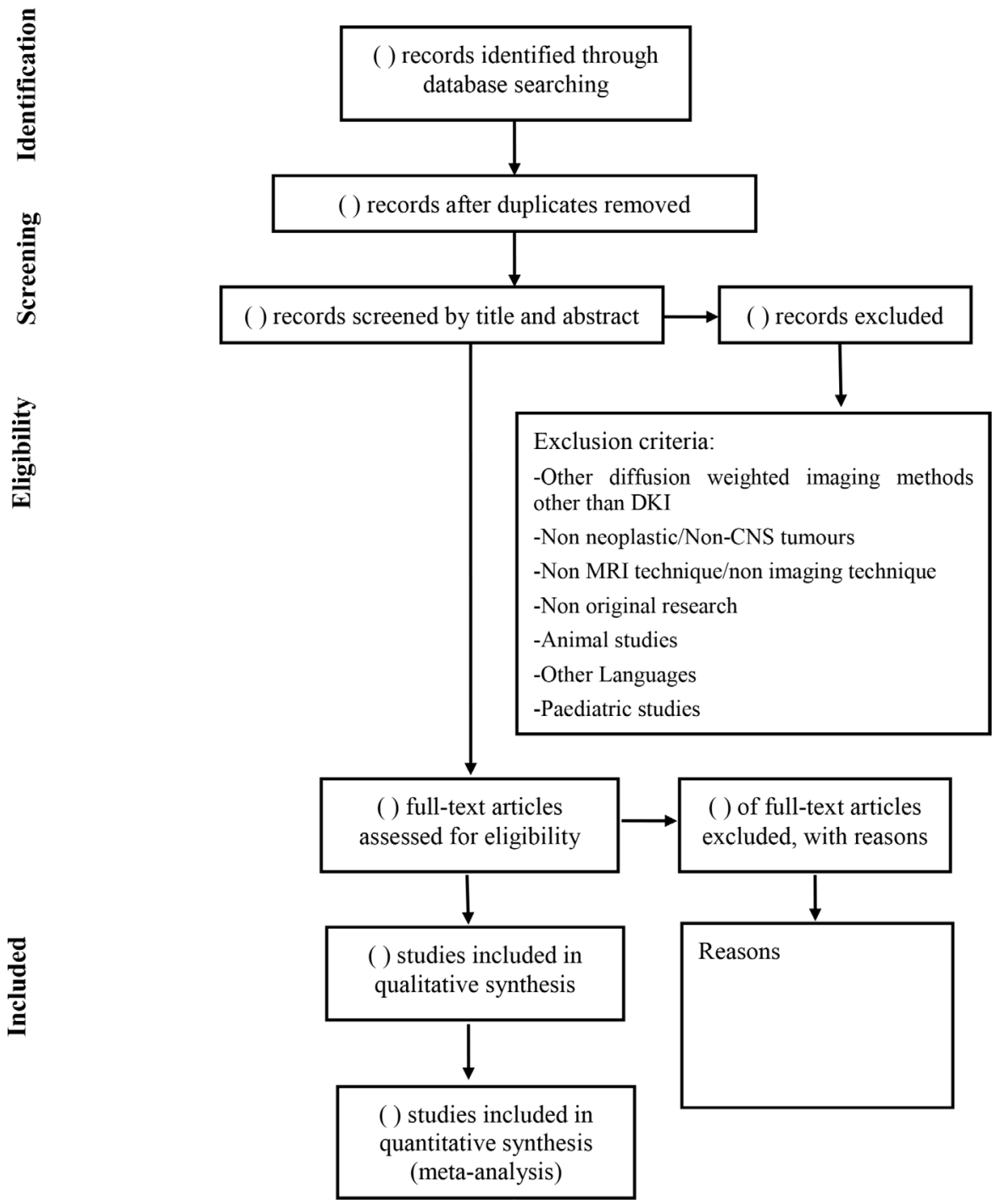

Figure 1 Flow diagram for search strategy. CNS, central nervous system; DKI, diffusional kurtosis imaging.

DWI-MRI studies, non-neoplastic conditions, non-glial tumours only, non-cerebral tumours and studies written in languages other than English, French or German.

\section{Search strategy for identification of studies}

A systematic literature search will be developed in July 2018 in four databases: PubMed, Medline via Ovid, Scopus and Embase using the keywords 'glioma' and 'diffusional kurtosis' by both medical subject heading and text words, without language restrictions. The suggested search syntax on PubMed and Medline is summarised in table 1 and the flow chart is illustrated in figure 1.

\section{Study selection for inclusion in the review}

Initially, studies identified by the literature search will be independently screened for primary eligibility by two authors, based on title and abstract. Full text of the primarily eligible studies will be independently assessed for final inclusion in the systematic review and meta-analysis by the same raters. Any disagreements will be resolved through discussions. Reasons for study exclusion will be stated clearly.

\section{QUALITY ASSESSMENT}

Eligible studies will be independently assessed according to the revised tool of quality assessment of diagnostic accuracy studies-2 tool $^{14}$ by two coauthors. Any disagreements will be resolved by consensus. The tool comprises four key domains which are: patient selection, index test, reference standard and patients' flow in the study and timing of the reference standard and the index test. Under each domain, risk of bias will be assessed through answers to signalling questions given in the tool, taking into account the review questions. Regarding the patient selection domain, retrospective studies will be considered to have high risk of bias, meanwhile low risk will be attributed to prospective studies. Regarding the index test domain, whether the neuroradiologist was blinded to the pathology during the image processing and region of interest drawing or not will determine the risk of bias; the neuroradiologists being blinded indicates low risk of bias. Histopathological results will serve as the reference standard. In the domain of flow and timing, unclear risk of bias will be ascertained if the interval between the 
index test and the reference standard is not mentioned in the study. Additionally, unclearness will be considered also if any patient was excluded from the analysis without relevant reasons. Concerns regarding applicability will be assessed in the first three domains only.

\section{DATA EXTRACTION AND MANAGEMENT}

Data extraction will be performed by two coauthors in a predesigned standardised sheet. Extracted data will include the following: first author name, publication year, type of study, details about the patient population, data acquisition techniques, image processing, postprocessing software, reference standard and diagnostic test accuracy results (true-positive, false-positive, true-negative and false-negative values). Any missing data will be requested from the related study authors.

\section{DATA SYNTHESIS AND ANALYSES}

We will construct $2 \times 2$ tables using reported number of true-positive, false-positive, false-negative and true-negative cases to calculate different indicators of diagnostic performance. A narrative synthesis will summarise the available evidence. Paired forest plots illustrating sensitivity and specificity with their $95 \%$ CIs will be built using the Review Manager software. We will use a bivariate random-effect model for data synthesis based on the assumption that studies are of sufficient heterogeneity in terms of study populations and study methodology. We will also report the number of true positives, false positives, true negatives and false negatives. A random-effect meta-analysis as well as aggregation of data using the hierarchical summary receiver operator characteristics will be pursued.

\section{Author affiliations}

${ }^{1}$ Lysholm Department of Neuroradiology, The National Hospital for Neurology and Neurosurgery UCL Hospitals NHS Trust, London, UK

${ }^{2}$ MRC Centre for Neuromuscular Diseases \& Centre for Rheumatology, University College London, London, UK

${ }^{3}$ Jockey Club School of Public Health and Primary Care, Faculty of Medicine, The Chinese University of Hong Kong, Hong kong, China

${ }^{4}$ NIHR CLAHRC North Thames, Department of Applied Health Research, University College London, London, UK

${ }^{5}$ Department of Global Health and Development, London School of Hygiene and Tropical Medicine, London, UK

${ }^{6}$ Department of Global Health Policy, Graduate School of Medicine, University of Tokyo, Tokyo, Japan

${ }^{7}$ Department of Brain Repair and Rehabilitation, Institute of Neurology, University College London, London, United Kingdom

Contributors Study and protocol design was undertaken by TY and SB. GA and ES will contribute to the literature search, data extraction and drafted the protocol manuscript. PMM and JSWK applied for PROSPERO registration. DY, JPG and ARG designed the data analyses strategy and will perform the statistical analysis of the study. PMM, DY, JSWK, JP-G, AR-G, TY and SB revised the manuscript for intellectual content. All authors approved the final version of the manuscript.

Funding PMM and SB are supported by the National Institute for Health Research (NIHR) Biomedical Research Centre (BRC) at University College London Hospitals (UCLH) NHS Foundation Trust and University College London (UCL). JPG and ARG's research is supported by the National Institute for Health Research (NIHR) Collaboration for Leadership in Applied Health Research and Care North Thames at Bart's Health NHS Trust (NIHR CLAHRC North Thames).

Disclaimer The views expressed are those of the authors and not necessarily those of the (UK) National Health Service (NHS), the NIHR or the (UK) Department of Health.

Competing interests None declared.

Patient consent Not required.

Provenance and peer review Not commissioned; externally peer reviewed.

Open access This is an open access article distributed in accordance with the Creative Commons Attribution Non Commercial (CC BY-NC 4.0) license, which permits others to distribute, remix, adapt, build upon this work non-commercially, and license their derivative works on different terms, provided the original work is properly cited, appropriate credit is given, any changes made indicated, and the use is non-commercial. See: http://creativecommons.org/licenses/by-nc/4.0/.

\section{REFERENCES}

1. Jiang R, Jiang J, Zhao L, et al. Diffusion kurtosis imaging can efficiently assess the glioma grade and cellular proliferation. Oncotarget 2015;6:42380-93.

2. Hempel JM, Bisdas S, Schittenhelm J, et al. In vivo molecular profiling of human glioma using diffusion kurtosis imaging. $J$ Neurooncol 2017;131:1-9.

3. Hempel JM, Schittenhelm J, Brendle C, et al. Effect of perfusion on diffusion kurtosis imaging estimates for in vivo assessment of integrated 2016 WHO glioma grades: a cross-sectional observational study. Clin Neuroradiol 2017:1-11.

4. Hempel JM, Bisdas S, Schittenhelm J, et al. Erratum to: In vivo molecular profiling of human glioma using diffusion kurtosis imaging. J Neurooncol 2017;131:103. (J Neuro-Oncol 2017; 131:93-101).

5. Bisdas S, Shen H, Thust S, et al. Texture analysis- and support vector machine-assisted diffusional kurtosis imaging may allow in vivo gliomas grading and IDH-mutation status prediction: a preliminary study. Sci Rep 2018;8:6108.

6. Tietze A, Hansen MB, Østergaard L, et al. Mean diffusional kurtosis in patients with glioma: initial results with a fast imaging method in a clinical setting. AJNR Am J Neuroradiol 2015;36:1472-8.

7. Van Cauter S, Veraart J, Sijbers J, et al. Gliomas: diffusion kurtosis MR imaging in grading. Radiology 2012;263:492-501.

8. Hempel JM, Schittenhelm J, Brendle C, et al. Histogram analysis of diffusion kurtosis imaging estimates for in vivo assessment of 2016 WHO glioma grades: A cross-sectional observational study. Eur J Radiol 2017:95:202-11.

9. Hempel JM, Schittenhelm J, Bisdas S, et al. In vivo assessment of tumor heterogeneity in WHO 2016 glioma grades using diffusion kurtosis imaging: Diagnostic performance and improvement of feasibility in routine clinical practice. J Neuroradiol 2018;45.

10. Falk Delgado A, Nilsson M, van Westen D, et al. Glioma grade discrimination with $\mathrm{mr}$ diffusion kurtosis imaging: a meta-analysis of diagnostic accuracy. Radiology 2018;287.

11. Shamseer L, Moher D, Clarke M, et al. Preferred reporting items for systematic review and meta-analysis protocols (PRISMA-P) 2015: elaboration and explanation. BMJ 2015;349:g7647.

12. Campbell J, Kulgar M, Carmody D, et al. Chapter 9: diagnostic test accuracy systematic reviews. In: Aromataris E, Munn Z, eds. Joanna Briggs Institute reviewer's manual: The Joanna Briggs Institute, 2017

13. Mclnnes MDF, Moher D, Thombs BD, et al. Preferred reporting items for a systematic review and meta-analysis of diagnostic test accuracy studies: the PRISMA-DTA Statement. JAMA 2018;319:388

14. Whiting PF, Rutjes AW, Westwood ME, et al. QUADAS-2: a revised tool for the quality assessment of diagnostic accuracy studies. Ann Intern Med 2011;155:529. 